\title{
Tilapia Invasion Impacts Trophic Position And Resource Use Of Commercially Harvested Piscivorous Fish In The Large Subtropical Pearl River
}

Fangmin Shuai ( $\nabla$ sfm@prfi.ac.cn)

Pearl River Fisheries Research Institute, Chinese Academy of Fishery Sciences https://orcid.org/0000-0002-0676-2431

Jie Li

Pearl River Fisheries Research Institute,CAFS,Guangzhou 510280, China

Sovan Lek

University de Toulouse-PaulSabatier, 118 route de Narbonne, 31062 Toulouse Cedex, France

\section{Research Article}

Keywords: Tilapia, Invasion, Stable isotope ratios, Trophic position, SIMMs

Posted Date: March 7th, 2022

DOI: https://doi.org/10.21203/rs.3.rs-1309813/v1

License: () (i) This work is licensed under a Creative Commons Attribution 4.0 International License. Read Full License 


\section{Abstract}

Species invasions pose a serious threat to native biodiversity and ecosystems. However, quantifying the impacts of invasive species has proven problematic. In this study, we quantified the trophic changes in freshwater food webs invaded by tilapia, using an extensive stable isotope dataset to compare uninvaded and invaded rivers downstream of the Pearl River, China. The trophic position of the widely distributed and locally economically important piscivorous culter fish (Erythroculter recurviceps), mandarin fish (Siniperca kneri), and catfish (Pelteobagrus fulvidraco and Pelteobagrus vachelli) decreased significantly in the invaded river compared to the uninvaded river. Our analysis indicated that the decrease in tropic position of these piscivorous fishes reflected a major reduction in the proportion of prey fish biomass as a result of tilapia invasion. Stable isotope mixing models (SIMMs) indicated that small fish in the diet of culter fish from the reference river (32.7\% small fish, $17 \%$ zooplankton) were replaced by lower trophic level zooplankton prey in the invaded river ( $35.7 \%$ zooplankton, $25.4 \%$ small fish), due to the presence of tilapia. Small fish in the diet of mandarin fish in the reference river (46.2\% small fish, $10.5 \%$ aquatic insects) was replaced by lower trophic level aquatic insect prey in the invaded river ( $20.3 \%$ aquatic insects, $29.9 \%$ small fish). Fish eggs in the diet of catfish from the reference river ( $25.0 \%$ fish eggs, $25.2 \%$ aquatic insects) were replaced by aquatic insects at a lower trophic level in the invaded river ( $43.5 \%$ aquatic insects, $4.8 \%$ fish eggs). The results of this study contributed to a growing body of evidence, showing that tilapia could modify trophic interactions, which had severe consequences in invaded ecosystems.

\section{Introduction}

Although freshwater ecosystems cover less than $1 \%$ of the earth's surface, they support extremely high levels of biodiversity and provide irreplaceable ecosystem services, including the provision of fish products (Lévéque et al. 2008). Freshwater ecosystems exhibit the highest species richness per unit area of all ecosystems (Balian et al. 2008). However, due to global change and human interference, aquatic ecosystem functioning has declined sharply (Jenkins 2003). Freshwater ecosystems are considered to be one of the most endangered ecosystems (Dudgeon et al. 2006) and have the highest extinction rates on Earth (Michelan et al. 2010).

The introduction of non-native fish, for instance for aquaculture or ornamental use, is widely recognized as a serious threat to the functioning of freshwater ecosystems (as a result of changes in species diversity or the extinction of native species due to competition for food resources) (Ehrenfeld 2010; Marr et al. 2010; Lockwood et al. 2011). A number of studies have reported that fish invasions can destabilize natural communities by altering food web structure and stability (Eby et al. 2006; Attayde et al. 2011; Goto et al. 2020). Although knowledge of how food web structure relates to invasive species establishment and how these disturbances drive changes in the trophic structure of native food webs remains poorly understood, it may potentially be an important aspect of global change (Wainright et al. 2021).

Quantitative predictions of trophic responses to species invasions remains challenging because the structure of food webs is variable and complex. In addition, invasive species often have broad diets, so they have the potential to interact with a wide variety of prey species (Theoharides and Dukes 2007 ; Polis and Winemiller 2013). Ongoing improvements in stable isotope technology have made it possible to detect the effects of invasive fish species on the structure of food webs, and further understand the subsequent impacts on ecosystem functioning (Cardinale 2012; Thompson et al. 2012; González-Bergonzoni et al. 2020). For instance, using stable isotopes, Vander - Zanden et al. (1999) first documented significant changes in the trophic positions of native lake trout (Salvelinus namaycush) after the invasion of two invasive fish species (Micropterus dolomieu and Ambloplites rupestris), caused by a diet shift from consuming littoral fish to pelagic zooplankton. The invasion of rainbow trout (Oncorhynchus mykiss) usurped terrestrial prey that fell into the stream, causing native Dolly Varden charr (Salvelinus malma) to shift their diet to insects, which resulted in the restructuring of stream and forest food webs (Baxter et al. 2004). Non-native species invasion has also been shown to increase food chain length in aquatic ecosystems and elevate contaminant levels (such as heavy metals) in top predators, not only reducing the stability of the ecosystem, but also threatening human health (Vörösmarty et al. 2010).

Tilapia, is the general name of all Tilapia spp., which are native to Africa, grow rapidly and show a range of biological responses to environmental conditions, such as disease resistance and increased environmental tolerance (Attayde et al. 2011). Tilapia has been introduced to at least 100 countries and has become one of the most important aquaculture species in the world (Martin et al. 2010; Grammer et al. 2012). However, these species have established viable wild populations in most tropical and subtropical environments (Zengeya et al. 2013). Wild populations were first reported from Australia in the 1970s (Ovenden et al. 2015), and now exist in at least 114 countries (Deines et al. 2016). Tilapia is currently one of the most widely distributed invasive fish, second only to Asian carps (Rutten et al. 2004). In China, tilapia was initially introduced into Guangdong province for aquaculture in 1957, following which, Tilapia culture developed rapidly in south China (Yao and Ye 2014; Fisheries and Fishery Administration Bureau of Ministry of Agriculture 2021).

The subtropical Pearl River is the largest river in south China and is over $2,400 \mathrm{~km}$ long. It is characterized by an average annual temperature of $23^{\circ} \mathrm{C}$, with very rich aquatic biological resources. The Pearl River supports high levels of biodiversity and is a popular area for global biodiversity research. The Pearl River supports 381 fish species, exhibits high endemism and a diverse gene pool (Lu 1990; Shuai et al. 2017). To restore and maintain fishery stocks, fishing moratoria, such as fishing bans during the spawning season, were introduced in 2010, and since 2018 fishing has not been allowed in the Pearl River Basin from March to June annually. One of the most serious ecological problems in the Pearl River is the invasion of tilapia in some tributaries (Gu et al. 2015; Shuai et al. 2019).

Although the top-down impacts of tilapia invasions on ecosystems has gained a lot of attention in recent years (Attayde et al. 2011; Russell et al. 2012; Córdova-Tapia et al. 2015), to date, it is still not fully understood how tilapia compete with native species for food resources and how this impacts the trophic structure of aquatic ecosystems, despite the ecological importance and urgency of this issue. Trophic position, which represents the food resource utilization characteristics of organisms at the local scale, is a key property linking ecosystem functioning and species invasion (Thompson et al. 2012). In addition, trophic position is the most intuitive and accurately measured ecological index of food web change. 
Therefore, in this study, we examined the relative trophic position of native piscivorous fishes to estimate the effects of invasive tilapia on food webs in the downstream sections of the Pearl River, China. Furthermore, we quantified how native piscivorous fish diets changed as tilapia invasion progressed, by using stable isotope mixing models (SIMMs). We selected the widely distributed and locally important commercially harvested culter fish (Hainan culter (Pelteobagrus vachelli), pelagic fish), mandarin fish (bigeye mandarin fish (Siniperca kneri), mesopelagic fish), and catfish (yellow catfish (Pelteobagrus fulvidraco) and darkbarbel catfish (Erythroculter recurviceps), demersal fish) as representative native piscivorous fish. By combining long-term abundance monitoring data and stable isotope analyses, we determined how invasion-induced trophic dynamics changed in downstream Pearl River food webs. It is crucial to understand the processes outlined in this study, in order to control non-native aquatic species, conserve the stability of freshwater ecosystems, and improve current conservation strategies in the Pearl River.

\section{Methods Study area}

The tributaries of the Dongjiang River downstream of the Pearl River were selected as the study river, and parallel tributaries of the Beijing River were selected as the reference river. The two parallel tributaries, the Dongjiang River and Beijiang River, have a similar geographical location and it is known from previous investigations and research that the environmental conditions are similar in both tributaries (see Appendix, Table S1 for details). However, there is a serious tilapia invasion in the Dongjiang River as a result of the aquaculture industry (Shuai et al. 2015; Gu et al. 2015), while the tilapia population in the Beijiang River is relatively small due to an underdeveloped aquaculture industry. Therefore, the Dongjiang and Beijiang Rivers provide a natural laboratory to study the impact mechanisms of tilapia invasion on river ecosystem functioning. A total of eight sampling sites (four in the invaded Dongjiang River and four in the reference Beijiang River) were established to provide sufficient samples (Fig. 1, Table 1).

Table 1

The coordinates of sampling sites along the Pearl River basin.

\begin{tabular}{|lllll|}
\hline Sites & Name & Coordinates & Width $(\mathrm{m})$ & Subordinate river \\
\hline S1 & Lubao & $112^{\circ} 53^{\prime} 23^{\prime \prime} \mathrm{E}, 23^{\circ} 20^{\prime} 53^{\prime \prime} \mathrm{N}$ & 791 & Beijiang \\
\hline S2 & Shijiao & $112^{\circ} 57^{\prime} 59^{\prime \prime} \mathrm{E}, 23^{\circ} 33^{\prime} 41^{\prime \prime} \mathrm{N}$ & 882 & Beijiang \\
\hline S3 & Qingyuan & $113^{\circ} 3^{\prime} 49^{\prime \prime} \mathrm{E}, 23^{\circ} 41^{\prime} 50^{\prime \prime} \mathrm{N}$ & 935 & Beijiang \\
\hline S4 & Lianjiang & $113^{\circ} 18^{\prime} 16^{\prime \prime} \mathrm{E}, 2^{\circ} \mathbf{1}^{\prime} 29^{\prime \prime} \mathrm{N}$ & 635 & Beijiang \\
\hline S5 & Hengli & $114^{\circ} 36^{\prime} 55^{\prime \prime} \mathrm{E}, 23^{\circ} 10^{\prime} 26^{\prime \prime} \mathrm{N}$ & 770 & Dongjiang \\
\hline S6 & Guzhu & $114^{\circ} 41^{\prime} 26^{\prime \prime} \mathrm{E}, 3^{\circ} 30^{\prime} 25^{\prime \prime} \mathrm{N}$ & 462 & Dongjiang \\
\hline S7 & Heyuan & $114^{\circ} 42^{\prime} 45^{\prime \prime} \mathrm{E}, 23^{\circ} 44^{\prime} 18^{\prime \prime} \mathrm{N}$ & 714 & Dongjiang \\
\hline S8 & Huangtian & $114^{\circ} 59^{\prime} 36^{\prime \prime} \mathrm{E}, 23^{\circ} 53^{\prime} 17^{\prime \prime} \mathrm{N}$ & 341 & Dongjiang \\
\hline
\end{tabular}

\section{Data Collection}

As fishing is prohibited in the entire Pearl River basin from March to June every year and there is no obvious winter season in the downstream stretches of the Pearl River basin, fish community samples were collected twice in spring (January and February), summer (July, August, September, and October) and autumn (November and December) at each sampling site from 2013 to 2020. Isotope sample collection was only carried out in the summer, to avoid the differences caused by seasons. Community sampling was carried out using a set of gillnets (length: $10 \mathrm{~m}$, height: $2.5 \mathrm{~m}$; mesh size: 20 mm), fishing hooks (length: 20 m, hooks: 50), and lobster pots (length: $15 \mathrm{~m}$, radius: $18 \mathrm{~cm}$ ) to overcome selectivity effects. All sampled fish were identified to species level and measured (total length, mm; wet weight, g)

For isotope sample collection, the white muscles of the fish were dissected from the upper side of the body and close to the dorsal fins, and put into a $5-\mathrm{mL}$ centrifuge tube. For the same fish species sampled at different locations, only adult samples were collected to reduce any possible confounding effects of life stage on isotopic values (Rennie et al. 2009). Phytoplankton and zooplankton were collected using a $250-$ mm zooplankton net. Aquatic insects such as mayflies were collected with a small hand-made net at the bottom of the river. Benthic snails and shrimp were placed in clean water for 24-48 hours, the shell was then removed, and the muscle tissue was placed into a 5-ml centrifuge tube. Any attached benthic algae and the leaves of aquatic plants were collected and washed, along with the attached sediment, in deionized water. Fish eggs and larvae were collected on spawning substrates, such as aquatic plants. All samples were stored in a mobile refrigerator at $-20^{\circ} \mathrm{C}$ and brought to the laboratory, where they were dried to constant weight at $60^{\circ} \mathrm{C}$, powdered and stored in a dryer. Each sample had at least six replicates and weighted between 0.5 and $1.0 \mathrm{mg}$.

\section{Stable Isotope Analyses}

Samples were placed in a drying tube and dried in an oven at a constant temperature of $105^{\circ} \mathrm{C}$ for $48 \mathrm{~h}$. The sample was weighed using a microbalance (Sartorius Service, Germany) with an accuracy of $0.001 \mathrm{~g}$ and wrapped in a tin capsule (volume: $48 \mu \mathrm{L}$, Thermo Fisher Scientific, US). The C and N isotope analysis was carried out on a Finnigan Delta V Advantage Isotope Ratio Mass Spectrometer (IRMS, Thermo Fisher Scientific, Inc., Waltham, Massachusetts, 
U.S.) and a Flash 2000 HT Elemental Analyzer (Thermo Fisher Scientific, Inc., Waltham, Massachusetts, U.S.) via a Conflo IV interface (Thermo Fisher Scientific, Inc., Waltham, Massachusetts, U.S.).

In this study, the trophic position of fishes was estimated relative to a primary "baseline" consumer, as basal trophic levels may vary between seasons and rivers (Cabana and Rasmussen 1996). Consumer trophic position was estimated using the formula: Trophic $_{\text {position }}$ consumer $=\left(\left(\delta 15 \mathrm{~N}_{\text {consumer }}-\right.\right.$

$\left.\left.\delta 15 N_{\text {baseline }}\right) / 3.4\right)+2$, where 3.4 is the assumed increase in $\delta 15 \mathrm{~N}$ per trophic level (Vander-Zanden and Rasmussen 1999). We chose Chironomids (Diptera: Chironomidae, Tabanidae, Stratiomyidae and Ephydridae) as our baseline consumer as they were abundant in all rivers sampled, and were collected in adequate numbers. Chironomids are also one of the main prey species of fish. Our analysis was based on the measurement of $\delta 15 \mathrm{~N}$ and $\delta 13 \mathrm{C}$ signatures from 684 samples from the eight study sites in two rivers.

Stomach contents were used to make preliminarily inferences on the diet of the representative fish. Representative fish individuals $(\mathrm{n}=30$ per site) were captured alive and measured to the closest $1 \mathrm{~cm}$ (total length, TL). Diet analysis was carried out based on the contents in the upper portion of the gut, to the first bend in the digestive tract. The stomach contents were removed from each individual and stored in $70 \%$ ethanol, before being analysed by stereomicroscope to check the frequency of occurrence of each source in the digestive tract. The frequency of occurrence is used to determine the composition of diet and the next isotopic analysis.

Changes in $\delta 13 \mathrm{C}$ or $\delta 15 \mathrm{~N}$ of an organism indicate a change in food source (Vander Zanden and Rasmussen 1999; Rennie et al. 2009). To compare the feeding ecology of the three piscivorous fish (culters, mandarin fish and catfish) in different rivers (the invaded Dongjiang River and uninvaded Beijiang River), we estimated the change of the potential contribution of food source using a Bayesian SIMM for R 3.5.3 (R Core Team 2019). SIMM is an upgrade of the SIAR model, which contains a slightly more sophisticated mixing model and uses Just Another Gibbs Sampler (JAGS) to run the model (Parnell et al. 2013). $113 \mathrm{C}$ and $\delta 15 \mathrm{~N}$ isotope ratios of the three piscivorous fish were put into the model as consumers. Means and SDs of $\delta 13 \mathrm{C}$ and $\delta 15 \mathrm{~N}$ information of small prey fishes, fish eggs, crustaceans, aquatic insects, zooplankton, snails, and aquatic plants were put into the model as source means and source standard deviations data. Other parameters in the model such as concentration and correction coefficients were set as default values (NULL). Gelman-Rubin convergence diagnostics were conducted to test if the model ran properly. The Gelman diagnostic values were all close to 1 , indicating that the model ran well. The posterior distribution for each source was reported as $95 \%$ credible intervals. The combination netting provided catch-per-unit-effort (CPUE, fish per net per day) estimates of relative densities of the piscivorous culter fish, mandarin fish, catfish, and their prey. All analyses were conducted using R Statistical Software version 3.3.1 (R Core Team 2019).

\section{Results}

\section{Fish community structure and variation}

A total of 10,623 individual fishes belonging to 74 taxa, 20 families, and seven orders were sampled during the present study in the invaded Dongjiang River. Of these, 66 were native and eight were non-native species. Cyprinids were most abundant, accounting for $59 \%$ of all the species caught. Of the eight nonnative species, tilapia was the most abundant, accounting for $13.24 \%$ of all individuals in the Dongjiang River (Table 2). The abundance of the other nonnative species was very low. A total of 10288 individuals belonging to 77 taxa, 17 families and seven orders were sampled in the reference Beijiang River. Of these, 71 were native and six were non-native species. Cyprinids were also the most abundant, accounting for $62 \%$ of all the species caught. The abundance of all the non-native species was very low, and tilapia abundance accounted for $4.84 \%$ of all individuals (Table 2 ).

Table 2 fish community structure in the Dongjiang River and Beijiang River

(E endemic to China; N native species; Non. Non-native species; RS River-sea migratory; RL River-lake migratory; SE Sedentawry; “+” indicates rare species) 


\begin{tabular}{|c|c|c|c|c|c|}
\hline \multirow[t]{2}{*}{ Species } & \multirow[t]{2}{*}{ English name } & \multicolumn{2}{|c|}{ Percentage (\%) } & \multirow[t]{2}{*}{ Feeding Habit } & \multirow[t]{2}{*}{ Category } \\
\hline & & Beijiang & Dongjian & & \\
\hline \multicolumn{6}{|l|}{ CYPRINIFORMES } \\
\hline \multicolumn{6}{|l|}{ Cyprinidae } \\
\hline Squalidus argentatus & Chub & 19.28 & 8.04 & 1 & $\mathrm{~N} ; \mathrm{RL}$ \\
\hline Hemiculter leucisculus & Common sawbelly & 15.05 & 17.63 & 0 & $\mathrm{~N} ; \mathrm{SE}$ \\
\hline Cirrhinus molitorella & Mud carp & 4.06 & 12.31 & $\mathrm{H}$ & $\mathrm{N} ; \mathrm{RL}$ \\
\hline Erythroculter recurviceps & Culter hainan & 3.70 & 0.85 & $\mathrm{P}$ & $\mathrm{N} ; \mathrm{SE}$ \\
\hline Pseudohemiculter dispar & & 3.59 & 0.37 & 0 & $\mathrm{~N} ; \mathrm{SE}$ \\
\hline Zacco platypus & Pale chub & 3.45 & 0.09 & 0 & $\mathrm{~N} ; \mathrm{SE}$ \\
\hline Squalidus wolterstorffi & Dot chub & 3.22 & 0.08 & 1 & $\mathrm{~N} ; \mathrm{RL}$ \\
\hline Squaliobarbus curriculus & Barbel chub & 2.91 & 1.52 & 0 & $\mathrm{~N} ; \mathrm{RL}$ \\
\hline Abbottina rivularis & Amur false gudgeon & 2.62 & 0.02 & 0 & $\mathrm{~N} ; \mathrm{SE}$ \\
\hline Cyprinus carpio & Carp & 1.92 & 1.51 & 0 & $\mathrm{~N} ; \mathrm{SE}$ \\
\hline Carassius auratus & Crucian & 1.82 & 2.55 & 0 & $\mathrm{~N} ; \mathrm{SE}$ \\
\hline Megalobrama terminalis & black amur bream & 1.69 & 5.47 & 0 & $\mathrm{~N} ; \mathrm{RL}$ \\
\hline Saurogobio dabryi & Longnose gudgeon & 1.94 & 4.79 & 1 & $\mathrm{~N} ; \mathrm{RL}$ \\
\hline Cirrhinus mrigala & Mrigal carp & 1.29 & 1.07 & 0 & NON;SE \\
\hline Hemibarbus labeo & & 1.29 & 0.71 & 0 & $\mathrm{~N} ; \mathrm{SE}$ \\
\hline Hemibarbus maculatus & & 1.20 & 1.14 & 0 & $\mathrm{~N} ; \mathrm{SE}$ \\
\hline Hypophthalmichthys molitrix & Silver carp & 1.14 & 1.50 & PL. & $\mathrm{N} ; \mathrm{RL}$ \\
\hline Opsariichthys bidens Günther & Chinese hooksnout carp & 1.07 & 0.93 & 1 & $\mathrm{~N} ; \mathrm{SE}$ \\
\hline Culter dabryi & Dashi culter & 1.05 & & $\mathrm{P}$ & $\mathrm{N} ; \mathrm{SE}$ \\
\hline Sarcocheilichthys parvus & & 0.91 & & 0 & $\mathrm{~N} ; \mathrm{SE}$ \\
\hline Ctenopharyngodon idellus & Grass carp & 0.80 & 0.81 & $\mathrm{H}$ & $\mathrm{N} ; \mathrm{RL}$ \\
\hline Aristichthys nobilis & Bighead carp & 0.75 & 0.38 & PL. & $\mathrm{N} ; \mathrm{RL}$ \\
\hline Platysmacheilus exiguus & & 0.71 & & 1 & $\mathrm{~N} ; \mathrm{RL}$ \\
\hline Rhodeus sinensis & Light's bitterling & 0.58 & & 0 & $\mathrm{~N} ; \mathrm{SE}$ \\
\hline Sinibrama wui & Bigeyes bream & 0.40 & 0.09 & 0 & $\mathrm{E} ; \mathrm{RL}$ \\
\hline Xenocypris davidi & Yellow tailed xenocypris & 0.35 & 2.28 & $\mathrm{H}$ & $\mathrm{N} ; \mathrm{RL}$ \\
\hline Onychostoma gerlachi & Largescale shoveljaw fish & 0.29 & & $\mathrm{H}$ & $\mathrm{N} ; \mathrm{SE}$ \\
\hline Hemiculterella wui & & 0.28 & & 0 & $\mathrm{E} ; \mathrm{SE}$ \\
\hline Puntius semifasciolatus & Chinese barb & 0.26 & & 0 & $\mathrm{~N} ; \mathrm{SE}$ \\
\hline Acrossocheilus beijiangensis & & 0.13 & & $\mathrm{H}$ & $\mathrm{N} ; \mathrm{SE}$ \\
\hline Osteochilus salsburyi & & 0.11 & 1.01 & 0 & $\mathrm{~N} ; \mathrm{SE}$ \\
\hline Parabramis pekinensis & White bream & 0.10 & 0.06 & $\mathrm{H}$ & $\mathrm{N} ; \mathrm{RL}$ \\
\hline Xenocypris argentea & silver xenocypris & 0.10 & 0.04 & & \\
\hline Culter alburnus & Topmouth culter & 0.08 & 0.57 & $P$ & $\mathrm{~N} ; \mathrm{SE}$ \\
\hline Erythroculter hypselonotus & Bigeyse culterfish & 0.07 & + & $\mathrm{p}$ & $\mathrm{N} ; \mathrm{SE}$ \\
\hline Megalobrama amblycephala & Wuchang fish & 0.05 & 0.02 & 0 & $\mathrm{~N} ; \mathrm{RL}$ \\
\hline Distoechodon tumirostris & Round mouth & 0.05 & 0.02 & $\mathrm{H}$ & $\mathrm{N} ; \mathrm{RL}$ \\
\hline Acheilognathus tonkinensis & Vietnamese bitterling & 0.03 & 0.66 & 0 & $\mathrm{~N} ; \mathrm{SE}$ \\
\hline Sinibrama melroseib & Hainan bream & 0.02 & 0.06 & 0 & $\mathrm{~N} ; \mathrm{SE}$ \\
\hline Mylopharyngodon piceus & Black carp & 0.02 & 0.01 & I & $\mathrm{N} ; \mathrm{RL}$ \\
\hline
\end{tabular}

Page 5/21 


\begin{tabular}{|c|c|c|c|c|c|}
\hline \multirow[t]{2}{*}{ Species } & \multirow[t]{2}{*}{ English name } & \multicolumn{2}{|c|}{ Percentage (\%) } & \multirow[t]{2}{*}{ Feeding Habit } & \multirow[t]{2}{*}{ Category } \\
\hline & & Beijiang & Dongjian & & \\
\hline Acrossocheilus parallens & & 0.02 & & $\mathrm{H}$ & $\mathrm{N} ; \mathrm{SE}$ \\
\hline Acrossocheilus labiatus & & 0.02 & & $\mathrm{H}$ & $\mathrm{N} ; \mathrm{SE}$ \\
\hline Acrossocheilus stenotaeniatus & & 0.02 & & $\mathrm{H}$ & $\mathrm{N} ; \mathrm{SE}$ \\
\hline Elopichthys bambusa & Yellow cheek carp & 0.02 & & $P$ & $\mathrm{~N} ; \mathrm{RL}$ \\
\hline Acheilognathus macropterus & Largefin bitterling & 0.02 & & 0 & $\mathrm{~N} ; \mathrm{SE}$ \\
\hline Rectoris posehensis & & 0.01 & & $\mathrm{H}$ & $\mathrm{N} ; \mathrm{SE}$ \\
\hline Cyprinus carpio var.specularis & Germany mirror carp & 0.01 & & 0 & $\mathrm{~N} ; \mathrm{SE}$ \\
\hline Labeo rohita & Roho labeo & 0.10 & & $\mathrm{D}$ & NON;SE \\
\hline Huigobio chenhsienensis & Huigobio gudgeon & & + & 1 & $\mathrm{~N} ; \mathrm{RL}$ \\
\hline Pseudogobio vaillanti & & & 0.08 & I & $\mathrm{N} ; \mathrm{RL}$ \\
\hline Acheilognathus chankaensis & Khanka spiny bitterling & & 0.26 & 0 & $\mathrm{~N} ; \mathrm{SE}$ \\
\hline Sarcocheilichthys nigripinnis & & & 0.15 & 0 & $\mathrm{~N} ; \mathrm{SE}$ \\
\hline Garra orientalis & Oriental sucking barb & & 0.04 & $\mathrm{H}$ & $\mathrm{N} ; \mathrm{SE}$ \\
\hline Pseudolaubuca sinensis & & & 0.03 & PL. & $\mathrm{N} ; \mathrm{SE}$ \\
\hline Pseudorasbora parva & Stone moroko & & 0.02 & 0 & $\mathrm{~N} ; \mathrm{SE}$ \\
\hline Tinca tinca & Tench & & 0.02 & 0 & NON;SE \\
\hline Spinibarbus denticulatus & & & 0.02 & 0 & $\mathrm{~N} ; \mathrm{RL}$ \\
\hline Gobiobotia meridionalis & & & 0.02 & 1 & $\mathrm{E} ; \mathrm{SE}$ \\
\hline Rhodeus spinalis Oshima & & & 0.01 & 0 & $\mathrm{~N} ; \mathrm{SE}$ \\
\hline Parasinilabeo assimilis & & & 0.01 & $\mathrm{H}$ & $\mathrm{N} ; \mathrm{SE}$ \\
\hline \multicolumn{6}{|l|}{ Cobitidae } \\
\hline Misgurnus anguillicaudatus & Oriental weatherfish & 4.06 & 3.53 & $\mathrm{D}$ & $\mathrm{N} ; \mathrm{SE}$ \\
\hline Micronoemacheilus pulcher & & 0.21 & 0.08 & $\mathrm{D}$ & $\mathrm{N} ; \mathrm{SE}$ \\
\hline Cobitis sinensis & Siberian spiny loach & 0.01 & 0.38 & 1 & $\mathrm{~N} ; \mathrm{SE}$ \\
\hline \multicolumn{6}{|l|}{ Homalopteridae } \\
\hline Vanmanenia hainanensis & & & 0.01 & 1 & E;SE \\
\hline \multicolumn{6}{|l|}{ PERCIFORMES } \\
\hline \multicolumn{6}{|l|}{ Cichlidae } \\
\hline Tilapia spp. & Tilapia & 4.84 & 13.24 & 0 & NON;SE \\
\hline \multicolumn{6}{|l|}{ Serranidae } \\
\hline Lateolabrax japonicus & Spotted sea bass & 1.20 & & 1 & $\mathrm{~N} ; \mathrm{RS}$ \\
\hline Siniperca kneri & Bigeye mandarinfish & 0.34 & 0.05 & $\mathrm{P}$ & $\mathrm{N} ; \mathrm{SE}$ \\
\hline Siniperca scherzeri & Spotted mandarinfish & 0.16 & & $\mathrm{P}$ & $\mathrm{N} ; \mathrm{SE}$ \\
\hline \multicolumn{6}{|l|}{ Channidae } \\
\hline Channa asiatica & Chinese snakehead & 0.02 & 0.27 & $\mathrm{P}$ & $\mathrm{N} ; \mathrm{SE}$ \\
\hline Channa maculata & Taiwan snakehead & 0.01 & 0.18 & $\mathrm{P}$ & $\mathrm{N} ; \mathrm{SE}$ \\
\hline Channa argus & Snakehead & 0.01 & & $\mathrm{P}$ & $\mathrm{N} ; \mathrm{SE}$ \\
\hline \multicolumn{6}{|l|}{ Eleotridae } \\
\hline Eleotris oxycephala & Sharphead sleeper & 0.49 & 0.20 & 1 & $\mathrm{~N} ; \mathrm{SE}$ \\
\hline Hypseleotris hainanensis & & & 0.01 & I & $\mathrm{N} ; \mathrm{SE}$ \\
\hline \multicolumn{6}{|l|}{ Gobiidae } \\
\hline Rhinogobius giurinus & Amur goby & 0.17 & 1.74 & 1 & $\mathrm{~N} ; \mathrm{SE}$ \\
\hline
\end{tabular}

Page 6/21 


\begin{tabular}{|c|c|c|c|c|c|}
\hline \multirow[t]{2}{*}{ Species } & \multirow[t]{2}{*}{ English name } & \multicolumn{2}{|c|}{ Percentage (\%) } & \multirow[t]{2}{*}{ Feeding Habit } & \multirow[t]{2}{*}{ Category } \\
\hline & & Beijiang & Dongjian & & \\
\hline Glossogobius giuris & Tongue goby & & 2.67 & 1 & $\mathrm{~N} ; \mathrm{SE}$ \\
\hline \multicolumn{6}{|l|}{ Anabantidae } \\
\hline Anabas testudineus & Climbing perch & & 0.01 & 0 & Non;SE \\
\hline \multicolumn{6}{|l|}{ Mastacembelidae } \\
\hline Mastacembelus armatus & Tire track eel & 0.41 & 0.55 & 1 & $\mathrm{~N} ; \mathrm{SE}$ \\
\hline \multicolumn{6}{|l|}{ SILURIFORMES } \\
\hline \multicolumn{6}{|l|}{ Bagridae } \\
\hline Pelteobagrus fulvidraco & Yellow catfish & 1.43 & 0.70 & 1 & $\mathrm{~N} ; \mathrm{SE}$ \\
\hline Pelteobagrus vachelli & Darkbarbel catfish & 1.27 & 1.48 & 1 & $\mathrm{~N} ; \mathrm{SE}$ \\
\hline Leiocassis crassilabris & Ussuri catfish & 1.07 & 0.02 & 1 & $\mathrm{~N} ; \mathrm{SE}$ \\
\hline Mystus guttatus & Spotted longbarbel catfish & 0.54 & 0.38 & 1 & $\mathrm{~N} ; \mathrm{SE}$ \\
\hline Leiocassis argentivittatus & Longitudinal catfish & 0.25 & 0.34 & 1 & $\mathrm{~N} ; \mathrm{SE}$ \\
\hline Mystus macropterus & Largefin longbarbel catfish & 0.01 & & I & $\mathrm{N} ; \mathrm{SE}$ \\
\hline Leiocassis virgatus & Striped catfish & & 0.37 & 1 & $\mathrm{~N} ; \mathrm{SE}$ \\
\hline \multicolumn{6}{|l|}{ Sisoridae } \\
\hline Glyptothorax fukiensis & & & 0.09 & I & $\mathrm{N} ; \mathrm{SE}$ \\
\hline \multicolumn{6}{|l|}{ Ictaluridae } \\
\hline letalurus Punetaus & Channel catfish & & 0.08 & 1 & NON;SE \\
\hline \multicolumn{6}{|l|}{ Clariidae } \\
\hline Clarias fuscus & Oriental catfish & 0.08 & 0.65 & 0 & $\mathrm{~N} ; \mathrm{SE}$ \\
\hline Clarias gariepinus & Fuscous catfish & 0.01 & 0.18 & 0 & NON;SE \\
\hline \multicolumn{6}{|l|}{ Siluridae } \\
\hline Silurus asotus & Catfish & 0.36 & 0.18 & $P$ & $\mathrm{~N} ; \mathrm{SE}$ \\
\hline \multicolumn{6}{|l|}{ Loricariidae } \\
\hline Hypostomus plecostomus & Suckermouth catfis & 0.05 & 0.05 & 0 & NON;SE \\
\hline \multicolumn{6}{|l|}{ CLUPEIFORMES } \\
\hline \multicolumn{6}{|l|}{ Clupeidae } \\
\hline Clupanodon thrissa & Chinese gizzard shad & 0.19 & & PL. & $\mathrm{N} ; \mathrm{RS}$ \\
\hline Konosirus punctatus & Dotted gizzard shad & 0.05 & & PL. & $\mathrm{N} ; \mathrm{RS}$ \\
\hline \multicolumn{6}{|l|}{ Engraulidae } \\
\hline Coilia grayii & Gray's grsnadier anchovy & 2.60 & 3.20 & 1 & $\mathrm{~N} ; \mathrm{SE}$ \\
\hline \multicolumn{6}{|l|}{ ANGUILLIFORMES } \\
\hline \multicolumn{6}{|l|}{ Anguillidae } \\
\hline Anguilla japonica & Japanese eel & & 0.03 & $P$ & $\mathrm{~N} ; \mathrm{RS}$ \\
\hline \multicolumn{6}{|l|}{ SYNBRANCHIFORMES } \\
\hline \multicolumn{6}{|l|}{ Synbranchidae } \\
\hline Monopterus albus & Finless eel & 0.08 & 0.06 & I & $\mathrm{N} ; \mathrm{RS}$ \\
\hline \multicolumn{6}{|l|}{ CHARACIFORMES } \\
\hline \multicolumn{6}{|l|}{ Anostomidae } \\
\hline Prochilodus scyofa & & 0.17 & 0.01 & 0 & NON;SE \\
\hline \multicolumn{6}{|l|}{ TETRAODONTIFORMES } \\
\hline
\end{tabular}




\begin{tabular}{|lllll|}
\hline Species & English name & \multicolumn{2}{l}{ Percentage (\%) } & Feeding Habit Category \\
\cline { 3 - 4 } & & \multicolumn{2}{l}{ Beijiang Dongjian } & \\
\hline Takifugu ocellatus & Ocellated puffer & + & I & N;RS \\
\hline
\end{tabular}

The main piscivorous fish in the Dongjiang and Beijiang River are culters, mandarin fish, and catfish. These fish are the most common and widely distributed fish in the current range occupied by tilapia. Moreover, the relative abundance of these three piscivorous fish in the local fish communities has remained stable over time in the Beijiang River, while culter fish (Rs, two-tailed $\mathrm{P}<0.01$, Fig. 2a) and catfish (Rs, two-tailed P< 0.005 , Fig. 2c) abundance decreased significantly in the invaded Dongjiang River. The number of prey fish species has not changed significantly over time in the invaded or reference rivers (Fig. 2d-2f). The relative densities of prey fish of the three piscivorous fish did not exhibit any significant changes over time in the Beijiang River, while all decreased significantly in the invaded Dongjiang River over time (Rs, two-tailed $\mathrm{P}<0.05$, Fig. $2 \mathrm{~g}-2 \mathrm{i}$ ).

For the three piscivorous fish, there was no significant difference in the number of prey fish species in the invaded Dongjiang River and the reference Beijing River (Fig. 3a). The catch data revealed that there were lower catch rates (fish per net per day) of prey fish for culter fish ( $t=6.705$, d.f. $=62, P<0.05$ ), mandarin fish $(t=5.009$, d.f. $=62, P<0.001)$, and catfish $(t=6.452$, d.f. $=62$, $P<0.05)$ in the invaded Dongjiang River compared with the reference Beijiang River (Fig. 3b, Table 3). 
Prey fish data of three piscivorous fish in invaded Dongjiang River and the reference Beijiang River

\begin{tabular}{|c|c|c|c|c|}
\hline Species & River & & Total no. of prey species(mean \pm sd) & Prey catch rate (grams per net per day, mean \pm sd) \\
\hline & \multicolumn{4}{|c|}{ Dongjiang } \\
\hline & & Hengli & $5.25(0.46)$ & $184.00(63.79)$ \\
\hline & & Guzhu & $6.13(0.64)$ & $196.75(65.60)$ \\
\hline & & Heyuan & $6.63(0.52)$ & $204.88(48.94)$ \\
\hline & & Huangtian & $5.88(0.64)$ & $138.00(50.55)$ \\
\hline & & Mean & $5.97(0.57)$ & $180.91(60.82)$ \\
\hline & \multicolumn{4}{|c|}{ Beijiang } \\
\hline & & Lubao & $7.00(0.93)$ & $317.13(105.29)$ \\
\hline & & Shijiao & $7.00(1.19)$ & $302.75(75.84)$ \\
\hline & & Qingyuan & $6.87(0.83)$ & $303.50(65.26)$ \\
\hline & & Lianjiang & $6.75(0.89)$ & $276.12(78.25)$ \\
\hline & & Mean & $6.91(0.12)$ & $299.88(79.84) *$ \\
\hline \multicolumn{5}{|c|}{ Mandarin fish } \\
\hline & \multicolumn{4}{|c|}{ Dongjiang } \\
\hline & & Hengli & $6.63(0.52)$ & $243.13(128.77)$ \\
\hline & & Guzhu & $6.50(0.76)$ & $175.50(80.46)$ \\
\hline & & Heyuan & $6.13(0.35)$ & 229.88 (99.40) \\
\hline & & Huangtian & $5.25(0.46)$ & 213.38 (95.67) \\
\hline & & Mean & $6.42(0.26)$ & $215.47(100.84)$ \\
\hline & \multicolumn{4}{|c|}{ Beijiang } \\
\hline & & Lubao & $7.00(0.76)$ & 483.88 (116.46) \\
\hline & & Shijiao & $7.25(0.71)$ & 334.25 (170.95) \\
\hline & & Qingyuan & $7.13(0.83)$ & 315.88 (79.78) \\
\hline & & Lianjiang & $6.50(0.53)$ & 306.88 (125.04) \\
\hline & & Mean & $6.97(0.33)$ & $369.56(141.87)^{* *}$ \\
\hline \multicolumn{5}{|l|}{ Catfish } \\
\hline & \multicolumn{4}{|c|}{ Dongjiang } \\
\hline & & Hengli & $4.5(0.76)$ & $129.38(26.65)$ \\
\hline & & Guzhu & $5.25(0.70)$ & $150.00(37.99)$ \\
\hline & & Heyuan & $4.88(0.64)$ & $134.38(45.34)$ \\
\hline & & Huangtian & $4.5(0.53)$ & $118.00(25.42)$ \\
\hline & & Mean & $4.78(0.36)$ & $132.94(35.11)$ \\
\hline & \multicolumn{4}{|c|}{ Beijiang } \\
\hline & & Lubao & $5.00(0.76)$ & 231.38 (95.89) \\
\hline & & Shijiao & $5.38(0.92)$ & $246.12(55.81)$ \\
\hline & & Qingyuan & $4.88(0.64)$ & $211.00(75.08)$ \\
\hline & & Lianjiang & $4.25(0.46)$ & $221.38(79.03)$ \\
\hline & & Mean & $4.88(0.47)$ & $227.47(75.07)^{*}$ \\
\hline
\end{tabular}

\section{Changes In Piscivorous Fish Food Webs After Tilapia Invasion}


We further investigated whether the abundance differences in prey fish between the invaded and reference rivers were consistent with differences in food webs between the rivers, as inferred from natural stable isotope distributions in river fish tissues. The trophic position of the three piscivorous fish also declined significantly in the invaded river compared to the reference river (Fig. 4a). The trophic position of culter fish averaged 3.94 in the invaded Dongjiang River, significantly lower than 4.64 in the reference Beijiang River $(t=-4.490$, d.f. $=46, p<0.05)$. The trophic position of mandarin fish averaged 4.14 in the invaded Dongjiang River, which was significantly lower than 4.93 in the reference Beijiang River $(t=-4.418, d . f .=46, p<0.01)$. The trophic position of catfish averaged 3.51 in the invaded Dongjiang River, which was significantly lower than 4.46 in the reference Beijiang River $(t=-3.977, d . f .=46, p<0.05)$. 
Table 4

Trophic position, $813 \mathrm{C}$ and mixing-model results

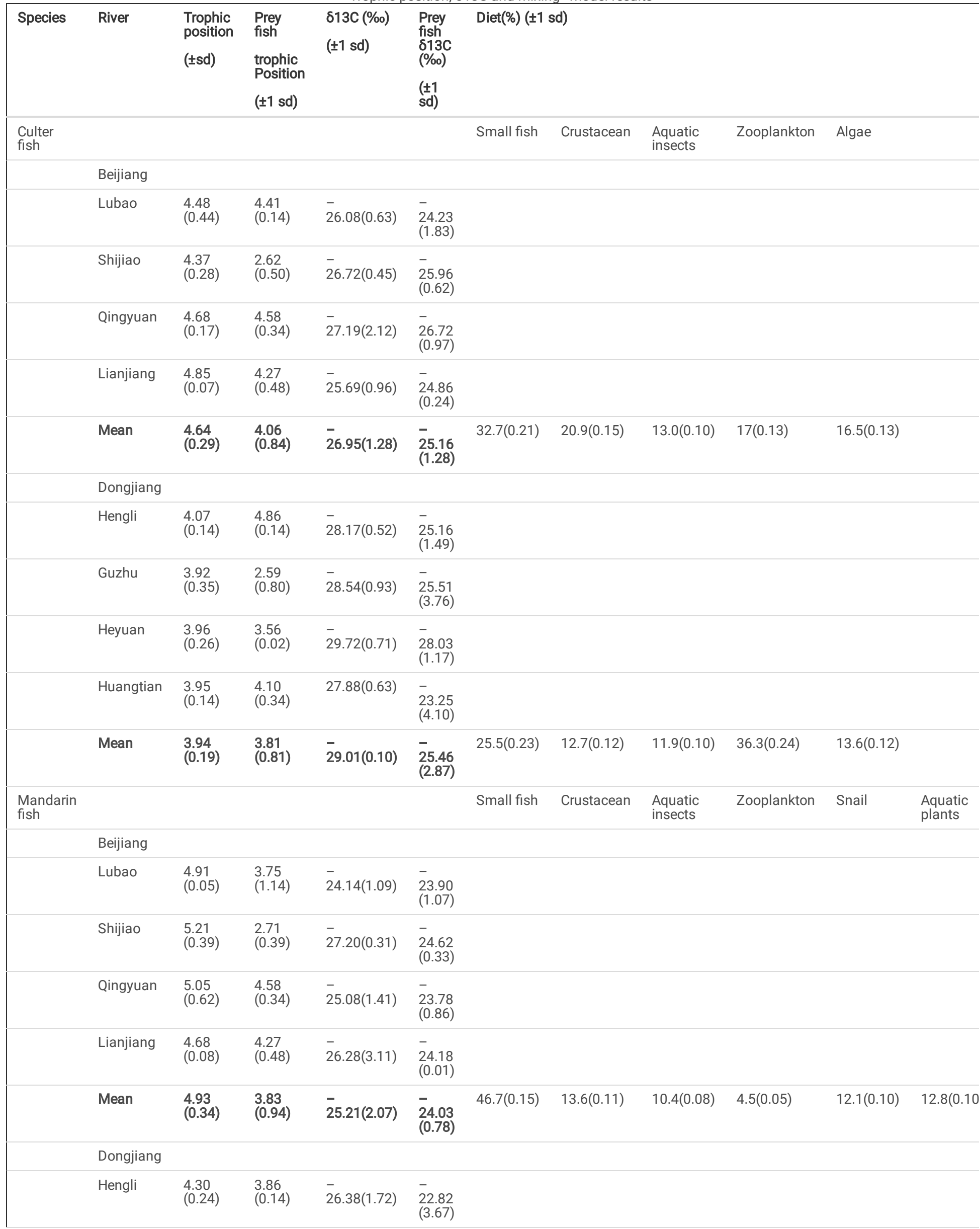




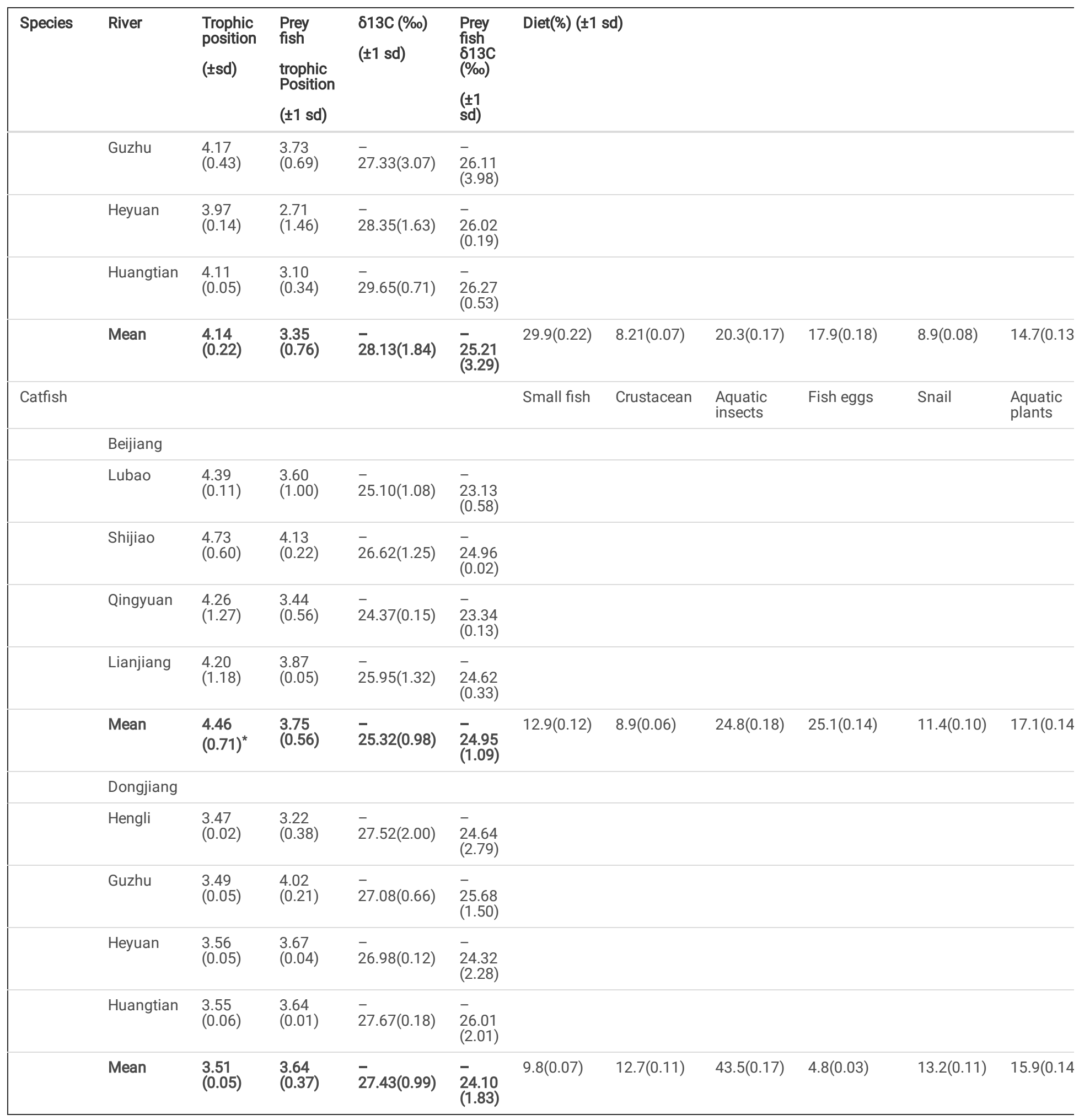

The $\delta 13 \mathrm{C}$ signatures provide additional evidence for differences in food webs between invaded and reference rivers. The $\delta 13 \mathrm{C}$ values in culter fish from the reference river averaged $-26.95 \%$, indicative of reliance on small prey fish, while $\delta 13 \mathrm{C}$ values in culter fish from invaded lakes was $-29.01 \%$, indicating greater use of zooplankton prey at lower trophic levels $(t=3.355$, d.f. $=46, P<0.01$, Table 4$)$. The $813 \mathrm{C}$ values in mandarin fish from the reference river averaged $25.21 \%$, indicating that small fish are also their main food source, while $\delta 13 \mathrm{C}$ values from the invaded lakes was $-28.13 \%$, indicating greater use of zooplankton and aquatic insects $(t=3.840$, d.f. $=46, P<0.05$, Table 4$)$. Similarly, $\delta 13 \mathrm{C}$ values in catfish from the reference river averaged $-25.32 \%$, while $\delta 13 \mathrm{C}$ values in catfish from invaded lakes was $-27.43 \%$, also indicating great differences in the use of food resources $(t=6.003, P<0.01$, Table 4$)$.

SIMMs using food source data from observations indicated that the diet of culter fish from the reference river averaged $32.7 \%$ small fish, compared with only $25.4 \%$ small fish and $36.3 \%$ zooplankton for culter fish from the invaded river (Fig. $5 a-5 b$ ). The diet of mandarin fish from the reference river averaged $46.2 \%$ small fish and $10.4 \%$ aquatic insects, compared with $29.9 \%$ small fish and $20.3 \%$ aquatic insects for mandarin fish in the invaded river (Fig. $5 \mathrm{c}-5 \mathrm{~d}$ ). SIMMs indicated that the diet of catfish from the reference river averaged $25.1 \%$ fish eggs and $24.8 \%$ aquatic insects, compared with only $4.8 \%$ fish eggs and $43.5 \%$ aquatic insects in the invaded river (Fig. 5e-5f). 


\section{Discussion}

Tilapia occur in more than 100 countries outside of their native range after tilapia has been introduced with joy for more than 60 years (Esselman et al. 2013). In 2014, tilapia was officially listed as one of the world's top 100 invasive species in the list of non-native invasive species in China (the third batch). In the present study, we found that tilapia invasion decreased the mean estimated trophic position of native top fish predators. Our analysis clearly demonstrated that this decrease in trophic position was solely due to the decline in prey fish biomass associated with tilapia invasion.

Tilapia have a preference for the same type of habitat as native fish and so the presence of tilapia displaced native fish from their preferred habitats. Tilapia invasion can reduce local biodiversity and result in the extinction of native fish species due to competitive replacement (Starling et al. 2002; Figueredo \& Giani 2005). Therefore, the establishment of tilapia has detrimental effects on aquatic food web structure in native habitats (Martin et al. 2010; Attayde et al. 2011; Russell et al. 2012). Fishes which are adapted to consume a diversity of foods often change their diets to overcome increased competition for food following species invasions (McMeans et al 2016; Wainright et al. 2021). These diet changes, such as switching from a specialist to a generalist diet or eating insects instead of fish, are reflected in the trophic structure of food webs.

This is the first study to clarify how the invasion of tilapia affects the feeding habits and trophic position of native species. There was strong evidence of a shift in diet composition and a decline in the trophic position of top fish predators in the invaded Dongjiang River related to changes in prey availability. The trophic position of culter fish, mandarin fish, and catfish in the invaded Dongjiang River, was significantly lower than in the reference Beijiang River. The diet of culter fish shifted from small fish ( $32.7 \%$ small fish, $17 \%$ zooplankton) to zooplankton ( $36.3 \%$ zooplankton, $25.5 \%$ small fish) in the invaded river. The diet of mandarin fish shifted from small fish (46.7\% small fish, $10.4 \%$ aquatic insects) to aquatic insects (20.3\% aquatic insects, $29.9 \%$ small fish) in the invaded river. The diet of catfish changed from fish eggs ( $25.1 \%$ fish eggs, $24.8 \%$ aquatic insects) to aquatic insects ( $43.5 \%$ aquatic insects, $4.8 \%$ fish eggs) in the invaded river.

This dietary shift was accompanied by a prolonged reduction in the abundance of native fish species. The sampling data showed that the relative densities of native prey fish decreased significantly over time in the invaded Dongjiang River. There has been a great deal of evidence to show that the increase of tilapia in rivers affects the CPUE of the fish community and native fish species (Gu et al. 2015), including the most abundant native species mud carp (Cirrhinus molitorella), black amur bream (Megalobrama terminalis), barbel chub (Squaliobarbus curriculus) and common sawbelly (Hemiculter leucisculus) (Shuai et al. 2019). The larvae of these fish are an important food source for top predators. A significant reduction in the CPUE of other commercially important species was also observed after the introduction of Nile tilapia in the North-eastern Brazil reservoir (Attayde et al. 2011). There is substantial overlap in diet between tilapia and native fishes in most tropical and subtropical habitats (Henson et al. 2016). In the current study, which spanned 9 years in the Pearl River, native fish densities decreased with increasing tilapia density. In particular, a progressive decrease in body size, such as fish plumpness, body length, and body weight, of native fishes coincided with the increasing prevalence of Nile tilapia (Shuai et al. 2019), and increased competition from Nile tilapia with local native species for food resources.

Trophic position stability is considered to be an important variable in the structural stability of food webs (Rennie et al. 2011; Thomsen et al. 2014). Analyzing trophic position variation can be helpful in detecting the effects of invasive fish species on the structure of food webs and understanding subsequent impacts on ecosystem functioning (Cardinale 2012; Thompson et al. 2012). Stable trophic positions of predators and prey are one component of stable food webs (Johnson et al. 2014), while trophic dispersion implicitly involves variability in trophic position. Tilapia invasion induced significant trophic dispersion, thereby disrupting trophic positions and destabilizing food webs in the Pearl River. We found that native top fish predators increasingly relied on zooplankton and aquatic insects as invasion progressed, which may have destabilized food webs and promoted their transition to tilapia dominance. Indeed, food web instability is a precursor to ecological state change (Rooney and McCann 2012), and biological invasions are known to yield alternative ecological states (Scheffer and Carpenter 2003), it is likely that these food web changes ultimately produced a new ecological regime (Wainright et al. 2021).

We found that invasive tilapia forced other fishes to increasingly rely on zooplankton and aquatic insect resources in the tropical river. These results demonstrated how invasive tilapia initiated disruption of native food webs via trophic displacement, and the study provided clear evidence that invasive predators can influence the dominant energy pathways of native predators, ultimately destroying ecosystem stability. The results of this study provided a basis for understanding and predicting the directional effects of invasive species on recipient food webs. Trophic changes due to fish invasion can also exhibit biotic homogenization with trophic downgrading (Singh 2021). For example, the invasion of lake trout (Salvelinus namaycush) increased fish diet variability, disrupted food webs by reorganizing macroinvertebrate communities, and displaced native fishes from their reference diets in the northern Rocky Mountains, USA (Wainright et al. 2021). The invasion of Dreissenid mussels, including the zebra mussel (Dreissena polymorpha) and quagga (Dreissena rostiformus bugensis) in the Great Lakes, caused commercially harvested native whitefish (Coregonus clupeaformis) to become more reliant on nearshore benthic production, changing the fundamental energy pathways in the lakes (Fear et al. 2017).

Therefore, the invasion of tilapia is bound to have a serious impact on the trophic position of native fish populations, and the negative impact of tilapia on native fisheries and ecosystems in southern China should not be underestimated. Protecting native fish populations often involves stopping the intentional introduction of non-native fish. The potential damage associated with invasive species has prompted recent efforts to predict the vulnerability of ecosystems to species invasions and prioritize them for management (Strassburg et al. 2020; McDonald-Madden et al. 2016). Ultimately, protecting entire landscapes from biological invasions may be required to sustain native biodiversity and ecosystems. This strategy may require stopping the introduction of invasive species, including non-native fish-stocking programs, and using innovative bio-surveillance monitoring techniques, such as environmental DNA (Evans et al. 2017), for early detection of potential invaders.

However, tilapia plays a very important role in the international market, ranking second in the global freshwater fish trade, second only to salmon and trout. Tilapia is one of the most internationally competitive aquaculture varieties in China, and it is also the species with the most potential for industrial development (Yao and ye 2014). In 2020, the global culture output of tilapia reached about 6.93 million tons. The huge demand for tilapia in the international

Page $13 / 21$ 
market has further expanded the breeding scale of tilapia in China. The production of tilapia in aquaculture in China reached 1.66 million tons in 2020 (Fisheries and Fishery Administration Bureau of Ministry of Agriculture 2021). For many years, China has been the world's largest tilapia producer and leading tilapia exporter (more than $60 \%$ of global tilapia exports), exporting to 80 countries or regions every year (Liao et al. 2020).

In spite of available regulatory approaches and guidelines to manage aquatic invasive species, fish invasions are increasing. The importance of the tilapia breeding industry, makes it difficult to control tilapia invasion, and it is neither realistic nor desirable to completely eradicate tilapia. To date, at least 10 tilapia species have been recorded in China, including New tilapia zillii, tilapia zillii, Mossambica tilapia (Oreochromis mossambicus), Nile tilapia (Oreochromis niloticus), Fushou tilapia (Mossambica tilapia $\times$ Nile tilapia), aureus tilapia (Oreochromis aureus), O'nei tilapia (aureus tilapia $\times$ Nile tilapia), blackchin tilapia (Sarotherodon melanotheron), Sarotherodon galilaeus and Tilapia rendalli (Yao and Ye 2014). As a result, feral tilapia have hybridized and introgressed in aquaculture settings before escaping to the wild. Reproductively viable hybrids have resulted, facilitating tilapia invasion. In order to better develop the aquaculture industry, germplasm improvement of tilapia is progressing rapidly. It is fairly well understood that hybrids, mixed hybrids and breeding, will lead to a general invasion success for most tilapia species. Therefore, after the serious ecological consequences caused by tilapia, the prevention and control of tilapia invasion is still a difficult problem.

In recent years, local governments have invested a lot of human and financial resources to protect and repair of the decline of fishery resources in rivers, such as annual proliferation and release activities, but these attempts have not been particularly successful. This was largely due to a lack of understanding regarding the mechanisms driving the decline in fishery resources. The results of the current study provided an initial insight into the decline of fishery resources in the Pearl River following tilapia invasion. At present, the most effective way to prevent invasion impacting fishery resources in the Dongjiang River is to be stringent regarding environmental isolation in pond culture, avoiding tilapia for release activities, and strictly controlling the growth range of this species. More stringent regulation of aquaculture activities and proactive fisheries management are required to avoid additional releases and further spread of tilapia in the region.

Understanding the consequences of invasive species on ecosystem functioning through changes in trophic interactions among species has received considerable interest over the past decade (Thébault 2003). Most of these studies have used stable isotopes to quantify changes in the trophic structure of communities (Cucherousset et al. 2012), as carbon $(\delta 13 \mathrm{C})$ and nitrogen $(\delta 15 \mathrm{~N})$ can provide an accurate quantitative method for the study of the changes of nutritional structure in aquatic ecosystems (Bearhop et al. 2004). Recent methodological developments have facilitated the quantification of multiple facets of the trophic structure of communities, such as isotopic diversity metrics, i.e. trophic niche width (TA), isotopic richness (IRic), isotopic evenness (IEve), isotopic divergence (IDiv), isotopic dispersion (IDis), and isotopic uniqueness (IUni) (Jackson et al. 2011; Cucherousset and Villéger 2015). These metrics were widely used to assess the effects of biological invasions on a multitude facets of food webs and ecosystem functioning at both local and global scales (Zambrano et al. 2010; Walsworth et al. 2013; Spurgeon et al. 2014; Sagouis et al. 2015).

While theoretical and methodological approaches have been recently developed, empirical studies are still needed to assess the effects of biological invasions on the trophic structure of recipient communities. The changes in food webs described in the current study have serious implications for native fish populations and food resources. An increased understanding of the interactions between tilapia and native fish is necessary for fishery management in many regions. Our findings emphasized the need to implement proactive control efforts to restore invaded ecosystems, particularly during colonization and early stages of establishment, to avoid food web disruptions that may be difficult to reverse. As tilapia is a commercially important species, its introduction cannot be banned, and so the strictest supervision of this species is required.

\section{Declarations}

Acknowledgements We are deeply grateful to the Heyuan and Qingyuan Detachment of the Guangdong Fishing Administrative Brigade for their assistance in the field. This work was supported by the National Natural Science Foundation of China (General Program No. 31870527) and the China-ASEAN Maritime Cooperation Fund (CAMC-2018F).

\section{References}

1. Attayde JL, Brasil J, Menescal RA (2011) Impacts of introducing nile tilapia on the fisheries of a tropical reservoir in north-eastern brazil. Fisheries Manag Ecol 18(6):437-443

2. Baxter CV, Fausch KD, Murakami M, Chapman PL (2004) Fish invasion restructures stream and forest food webs by interrupting reciprocal prey subésidies. Ecology 85(10):2656-2663

3. Balian EV, Segers H, Lévèque C, Martens K (2008) The freshwater animal diversity assessment: an overview of the results. Hydrobiologia 595:627-637

4. Cabana G, Rasmussen JB (1996) Comparison of aquatic food chains using nitrogen isotopes. PNAS 93:10844-10847

5. Cardinale BJ, Duffy JE, Gonzalez A, Hooper DU, Perrings C, Venail P, Narwani A, Mace GM, Tilman D, Wardle DA, Kinzig AP, Daily GC, Loreau M, Grace JB, Larigauderie A, Srivastava DS, Naeem S (2012) Biodiversity loss and its impact on humanity. Nature 486:5967)

6. Córdova-Tapia F, Contreras M, Zambrano L (2015) Trophic niche overlap between native and non-native fishes. Hydrobiologia 746(1):291-301)

7. Cucherousset J, Blanchet S, Olden JD (2012) Non-native species promote trophic dispersio of food webs. Front Ecol Environ 10(8):406-408)

8. Deines AM, Wittmann ME, Deines JM, Lodge DM (2016) Trade offs among Ecosystem Services Associated with Global Tilapia Introductions. Rev Fish Sci Aquac 2(24):178-191

9. Dudgeon D, Arthington AH, Gessner MO, Kawabata Z, Knowler D, Lévèque C, Naiman RJ, Prieur-Richard A, Soto D, Stiassny MLJ, Sullivan CA (2006) Freshwater biodiversity: importance, threats, status and conservation challenges. Biol Rev 81:163-182) 
10. Eby LA, Roach WJ, Crowder LB, Stanford JA (2006) Effects of stocking-up freshwater food webs. Trends Ecol Evol 21(10):576-584)

11. Ehrenfeld JG (2010) Ecosystem consequences of biological invasions. Annu Rev Ecol Evol Syst 41:59-80

12. Esselman PC, Schmitter-Soto JJ, Allan JD (2013) Spatiotemporal dynamics of the spread of African tilapias (Pisces: Oreochromis spp.) into rivers of northeastern Mesoamerica. Biol Invasions 15(7):1471-1491

13. Evans NT, Shirey PD, Wieringa JG, Mahon AR, Lamberti GA (2017) Comparative cost and effort of fish distribution detection via environmental DNA analysis and electrofishing. Fisheries 42:90-99

14. Fear SA, Rennie MD, Dunlop ES (2017) Broad shifts in the resource use of a commercially harvested fish following the invasion of dreissenid mussels. Ecology 98(6):1681-1692

15. Figueredo CC, Giani A (2005) Ecological interactions between Nile tilapia (Oreochromis niloticus, L) and the phytoplanktonic community of the Furnas Reservoir (Brazil). Freshwater Biol 50:1391-1403

16. Fisheries and Fishery Administration Bureau of Ministry of Agriculture 2021 (2021) China Fisheries Statistical Yearbook. China Agricultural Publishing House

17. Fry B (2006) Stable Isotope Ecology. Springer Science+Business Media, LLC

18. González-Bergonzoni I, Silva I, Mello S, Brugnoli E, Tesitore G, Vidal N, Naya DE (2020) Evaluating the role of predatory fish controlling the invasion of the asian golden mussel limnoperna fortunei in a subtropical river. J Appl Ecol 57(4):717-728

19. Goto D, Dunlop ES, Young JD, Jackson DA (2020) Shifting trophic control of fishery-ecosystem dynamics following biological invasions. Ecol Appl 30(8):e02190

20. Gu D, Ma G, Zhu Y, Xu M, Luo D, Li Y, Wei H, Mu X, Luo J, Hu Y (2015) The impacts of invasive Nile tilapia (Oreochromis niloticus) on the fisheries in the main rivers of Guangdong Province, China. Biochem Syst Ecol 59:1-7

21. Grammer GL, Slack WT, Peterson MS, Dugo MA (2012) Nile tilapia Oreochromis niloticus (Linnaeus, 1758) establishment in temperate Mississippi, USA: Multi-year survival confirmed by otolith ages. Aquat Invasions 7:367-376

22. Henson MN, Aday DD, Rice JA, Layman CA (2016) Assessing the influence of tilapia on sport species in north carolina reservoirs. T Am Fish Soc 147(2):1248-1254

23. Jenkins M (2003) Prospects for biodiversity. Science 302:1175-1177

24. Johnson S, Domínguez-García V, Donetti L, Muñoz MA (2014) Trophic coherence determines food-web stability. PNAS 111:17923-17928

25. Lévèque C, Oberdorff T, Paugy D, Stiassny MLJ, Tedesco PA (2008) Global diversity of fish (Pisces) in fresh water. Hydrobiologia 595:545-567

26. Lockwood JL, Hoopes MF, Marchetti MP (2011) Non-natives: plusses of invasion ecology. Nature 475(7354):36-36

27. Lu KX (1990) Pearl River Fishery Resources. Guangdong Science and Technology Press, Guanghzou, pp 91-121

28. Leprieur F, Beauchard O, Blanchet S, Oberdorff T, Brosse S (2008) Fish invasions in the world's river systems: when natural processes are blurred by human activities. PLoS Biol 6:e28)

29. Marr SM, Marchetti MP, Olden JD, García-Berthou E, Morgan DL, Arismendi I, Day JA, Griffiths CL, Skelton PH (2010) Freshwater fish introductions in mediterranean-climate regions: are the recommonalities in the conservation problem? Divers Distrib 16:606-619

30. Mcdonald-Madden E, Sabbadin R, Game ET, Baxter P, Chadès I, Possingham HP (2016) Using food-web theory to conserve ecosystems. Nat Commun 7:10245

31. Martin CW, Valentine MM, Valentine JF (2010) Competitive interactions between invasive Nile tilapia and native fish: the potential for altered trophic exchange and modification of food webs. PLoS ONE 5(12):e14395

32. McMeans BC, Mccann KS, Tunney TD, Fisk A (2016) The adaptive capacity of lake food webs: From individuals to ecosystems. Ecol Monogr 86:4-19

33. Ovenden JR, Macbeth GM, Pope L, Thuesen P, Street R, Broderick D (2015) Translocation between freshwater catchments has facilitated the spread of tilapia in eastern australia. Biol Invasions 17(2):637-650

34. Parnell AC, Phillips DL, Bearhop S, Semmens BX, Ward EJ, Moore JW, Jackson AL, Inger R (2013) Bayesian stable isotope mixing models. Environmetrics 24(6):387-399

35. Polis GA, Strong DR (1996) Food web complexity and community dynamics. Am Nat 147:813-846

36. Polis GA, Winemiller KO (2013) Food Webs: Integration of Patterns \& Dynamics. Springer Science \& Business Media

37. Rennie MD, Sprules WG, Johnson TB (2009) Resource switching in fish following a major food web disruption. Oecologia 159:789-802)

38. Rennie MD, Strecker AL, Palmer ME (2011) Bythotrephes invasion elevates trophic position of zooplankton and fish: implications for contaminant biomagnification. Biol Invasions 13:2621-2634)

39. Russell DJ, Thuesen PA, Thomson FE (2012) A review of the biology, ecology, distribution and control of Mozambique tilapia, Oreochromis mossambicus (Peters 1852) (Pisces: Cichlidae) with particular emphasis on invasive Australian populations. Rev Fish Biol Fisher 22:533-554)

40. Rooney N, McCann KS (2012) Integrating food web diversity, structure and stability. Trends Ecol Evol 27:40-46

41. Rutten MJM, Komen H, Deerenberg RM, Siwek M, Bovenhuis H (2004) Genetic characterization of four strains of Nile tilapia (Oreochromis niloticus L.) using microsatellite markers. Anim Genet 35:93-97)

42. R Core Team ( (2019) ) R: A language and environment for statistical computing. R Foundation for statistical Computing, Vienna, Austria. URL https://www.R-project.org/

43. Singh AK (2021) State of aquatic invasive species in tropical India: An overview. Aquat Ecosyst health 2(24):13-23 
44. Sagouis A, Cucherousset J, Villéger S, Santoul F, Boulêtreau S (2015) Non-native species modify the isotopic structure of freshwater fish communities across the globe. Ecography 38:979-985

45. Starling F, Lazzaro X, Cavalcanti C, Moreira R (2002) Contribution of omnivorous tilapia to eutrophication of a shallow tropical reservoir: evidence from a fish kill. Freshw Biol 147:2443-2452

46. Strassburg BBN, Iribarrem A, Beyer HL, Cordeiro CL, Crouzeilles R, Jakovac CC, Junqueira AB, Lacerda E, Latawiec AE, Balmford A (2020) Global priority areas for ecosystem restoration. Nature 586:724-729

47. Scheffer M, Carpenter SR (2003) Catastrophic regime shifts in ecosystems: Linking theory to observation. Trends Ecol Evol 18:648-656

48. Shuai FM, Li XH, Li YF, Li J, Yang JP, Lek S (2015) Forecasting the invasive potential of Niletilapia (Oreochromis niloticus) in a large subtropical river using an univariate approach. Fundam Appl Lim 187:165-176)

49. Shuai FM, Li XH, Chen FC, Li YF, Lek S (2017) Spatial patterns of fish assemblages in the Pearl River, China: environmental correlates. Fundam Appl Lim 189(4):329-340)

50. Shuai FM, Li XH, Liu QF, Zhu SL, Wu Z, Zhang YQ (2019) Nile tilapia (Oreochromis niloticus) invasions disrupt the functional patterns of fish community in a large subtropical river in China. Fisheries Manag Ecol 26(6):578-589)

51. Thompson RM, Brose U, Dunne JA, Hall RO, Hladyz S, Kitching RL, Martinez ND, Rantala H, Romanuk TN, Stouffer DB, Tylianakis JM (2012) Food webs: reconciling the structure and function of biodiversity. Trends Ecol Evol 27:689-697

52. Theoharides KA, Dukes JS (2007) Plant invasion across space and time: Factors affecting nonindigenous species success during four stages of invasion. New Phytol 176:256-273

53. Thomsen MS, Byers JE, Schiel DR, Bruno JE, Olden JD, Wernberg T, Silliman BR (2014) Impacts of marine invaders on biodiversity depend on trophic position and function similarity. Mar Ecol Prog ser 495:39-47)

54. Vander-Zanden MJ, Rasmussen JB (1999) Primary consumer $\delta 15 \mathrm{~N}$ and $\delta 13 \mathrm{C}$ and the trophic position of aquatic consumers. Ecology $80: 1395-1404$

55. Vander-Zanden MJ, Rasmussen JB (2001) Variation in d15N and d13C trophic fractionation: implications for aquatic food web studies. Limnol Oceanogr 46:2061-2066

56. Vörösmarty CJ, McIntyre PB, Gessner MO, Dudgeon D, Prusevich A, Green P, Glidden S, Bunn SE, Sullivan CA, Reidy-Liermann C, Davies PM (2010) Global threats to human water security and river biodiversity. Nature 467:555-561

57. Wainright CA, Muhlfeld CC, Elser JJ, Bourretd SL, Devlina SP (2021) Species invasion progressively disrupts the trophic structure of native food webs. PNAS 118(45):e2102179118

58. Yao GC, Ye W (2014) New technology of ecological and efficient culture of tilapia. China Ocean Press

59. Zengeya TA, Robertson MP, Booth AJ, Chimimba CT (2013) Ecological niche modeling of the invasive potential of Nile tilapia Oreochromis niloticus in African river systems: Concerns and implications for the conservation of indigenous congenerics. Biol Invasions 15:1507-1521

\section{Figures}




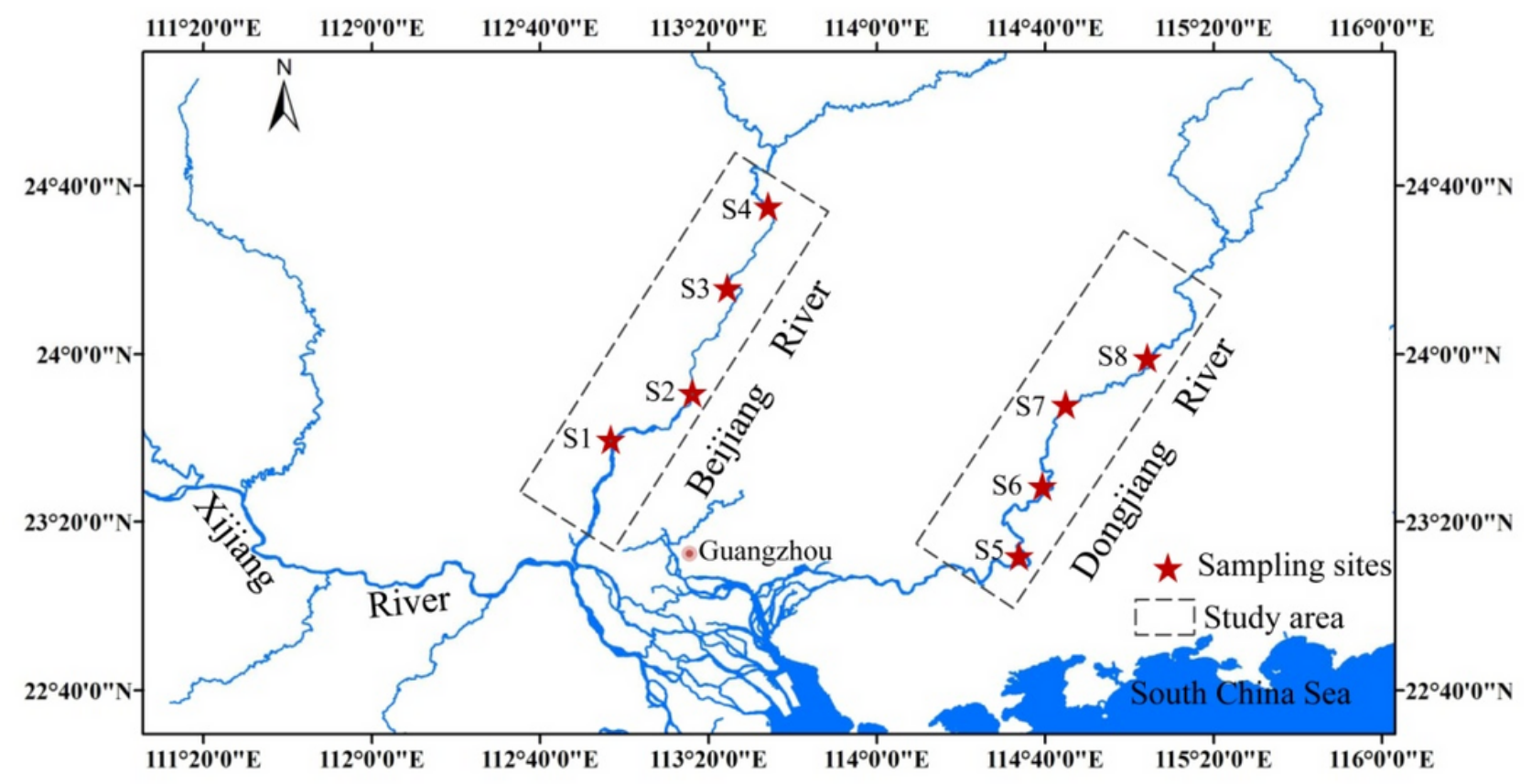

Figure 1

Sampling sites. 
(a) Culter fish

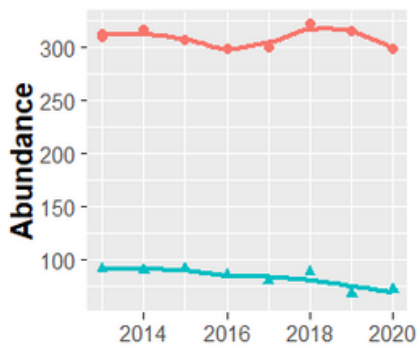

(d) Culter fish

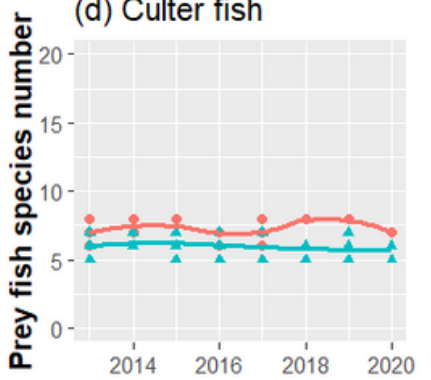

(g) Culter fish

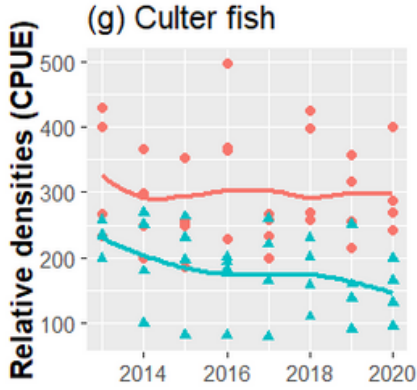

(b) Mandarin fish

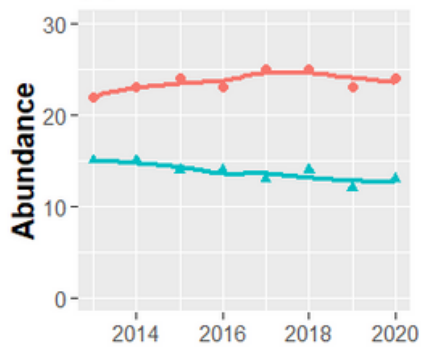

river

$\rightarrow$ Beijing

- Dongjiang

- Dongjiang

e) Mandarin fish

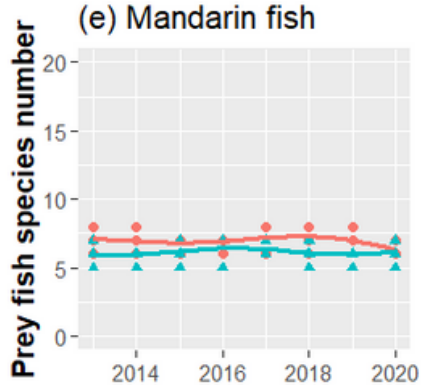

(h) Mandarinfish

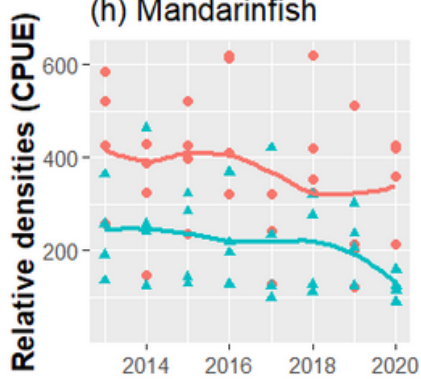

river (c) Catfish
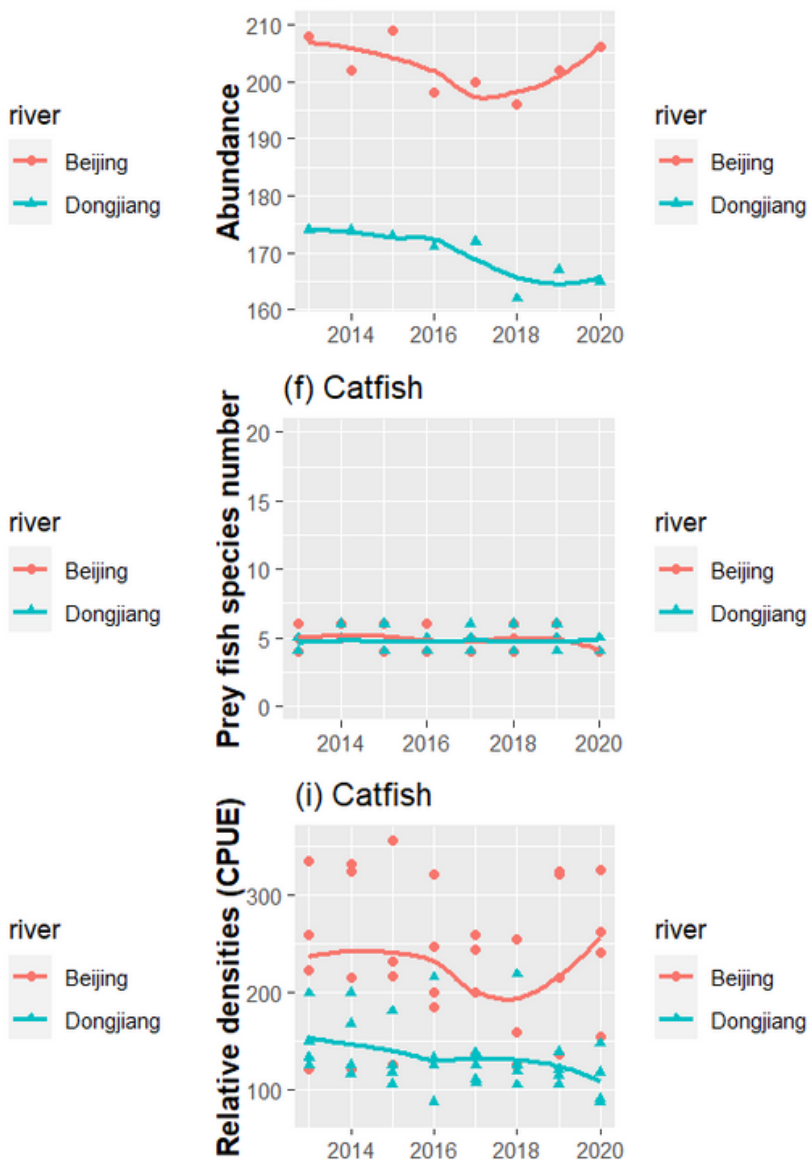

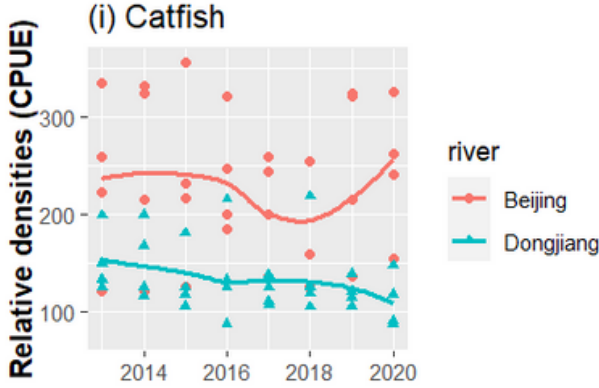

Figure 2

Temporal dynamics of three piscivorous fish and their prey fish. (a) abundance of culter fish, (b) abundance of mandarin fish, (c) abundance of catfish, (d) species number of prey fish of culter fish (e) species number of prey fish of mandarin fish, (f) species number of prey fish of catfish, (g) relative densities (CPUE, g per net per day) of prey fish of culter fish, (h) relative densities (CPUE) of prey fish of mandarin fish, (i) relative densities (CPUE) of prey fish of catfish in the Dongjiang River and Beijiang River from 2013 to 2020 (culter fish Erythroculter recurviceps, mandarin fish Siniperca kneri, catfish Pelteobagrus fulvidraco and Pelteobagrus vachell). 
(a)
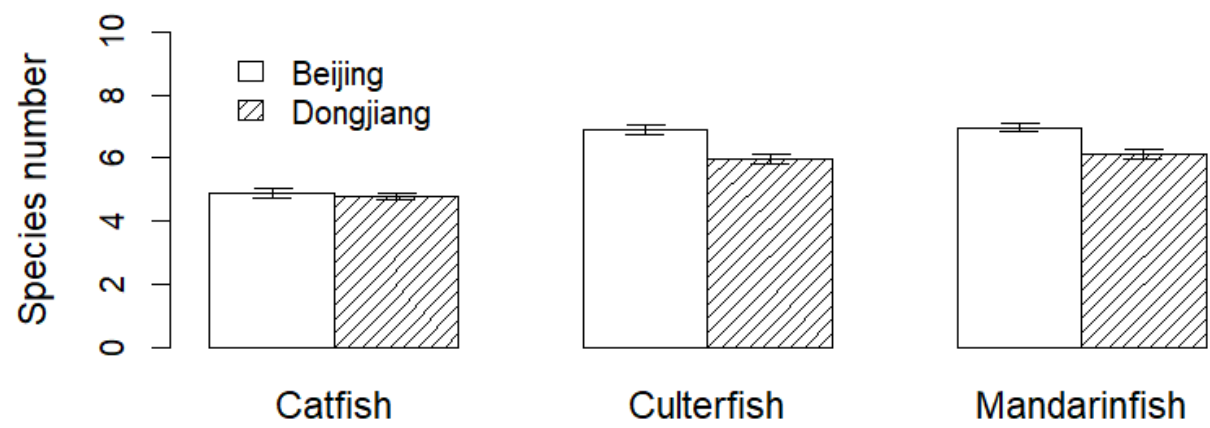

Mandarinfish

(b)
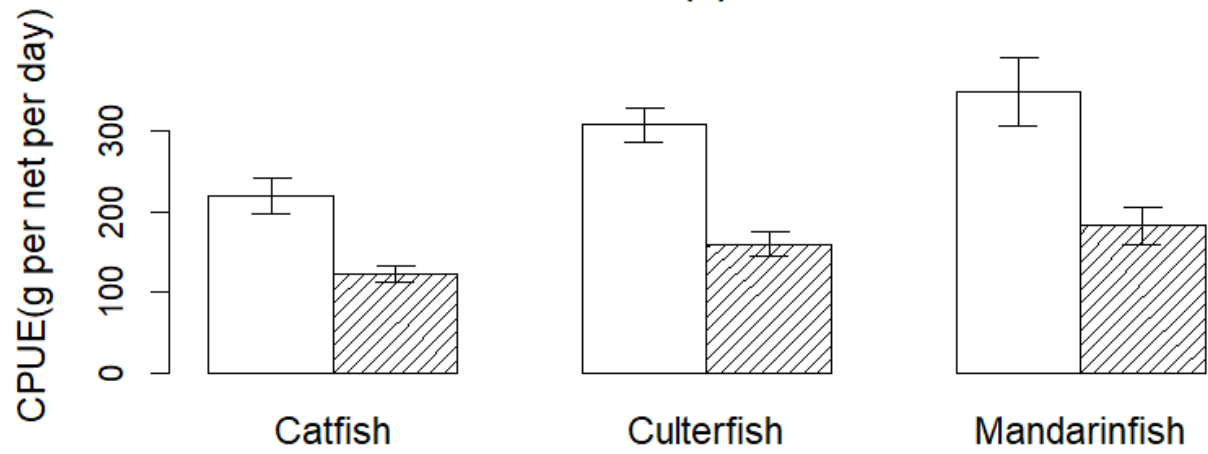

Figure 3

Prey fish data of the three piscivorous fish from the invaded and reference rivers. (a) Comparison the number of prey fish species, (b) Comparison of the relative densities (CPUE, g) of prey fish caught in each net each day. 
(a)
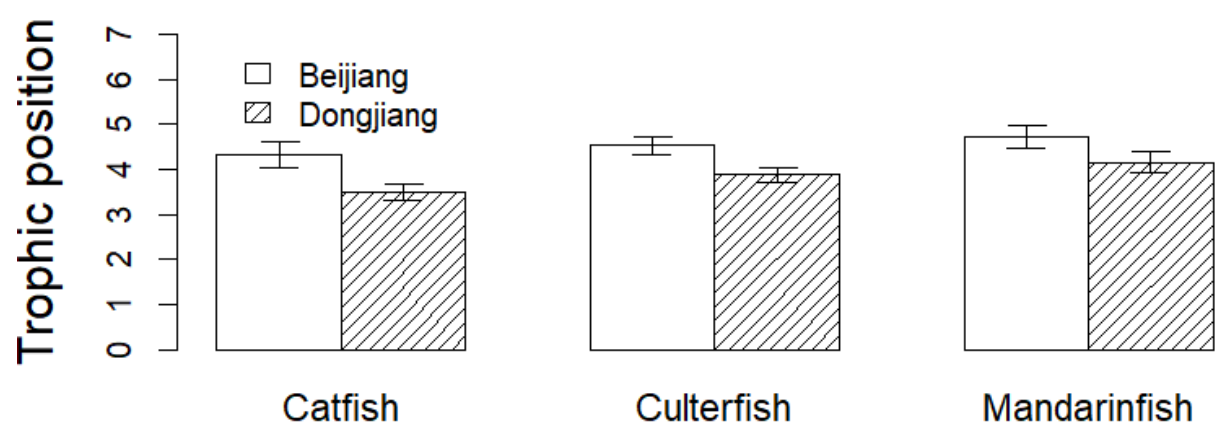

(b)
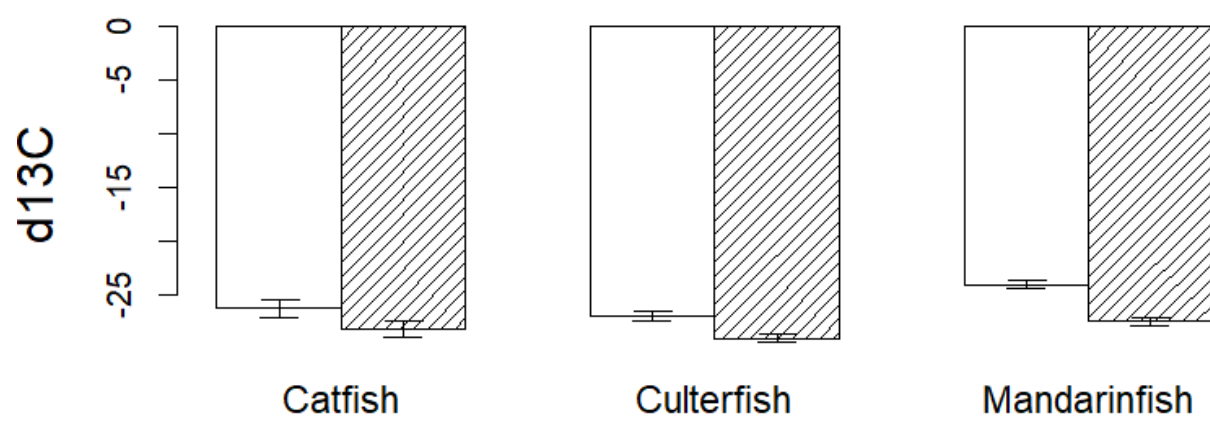

Mandarinfish

\section{Figure 4}

Trophic position and $\delta^{13} \mathrm{C}$ values. (a) Comparison of mean trophic position of piscivorous culter fish, mandarin fish, and catfish from invaded and reference lakes. (b) Comparison of mean $\delta 13 \mathrm{C}$ values of piscivorous culter fish, mandarin fish, and catfish from invaded and reference rivers. 

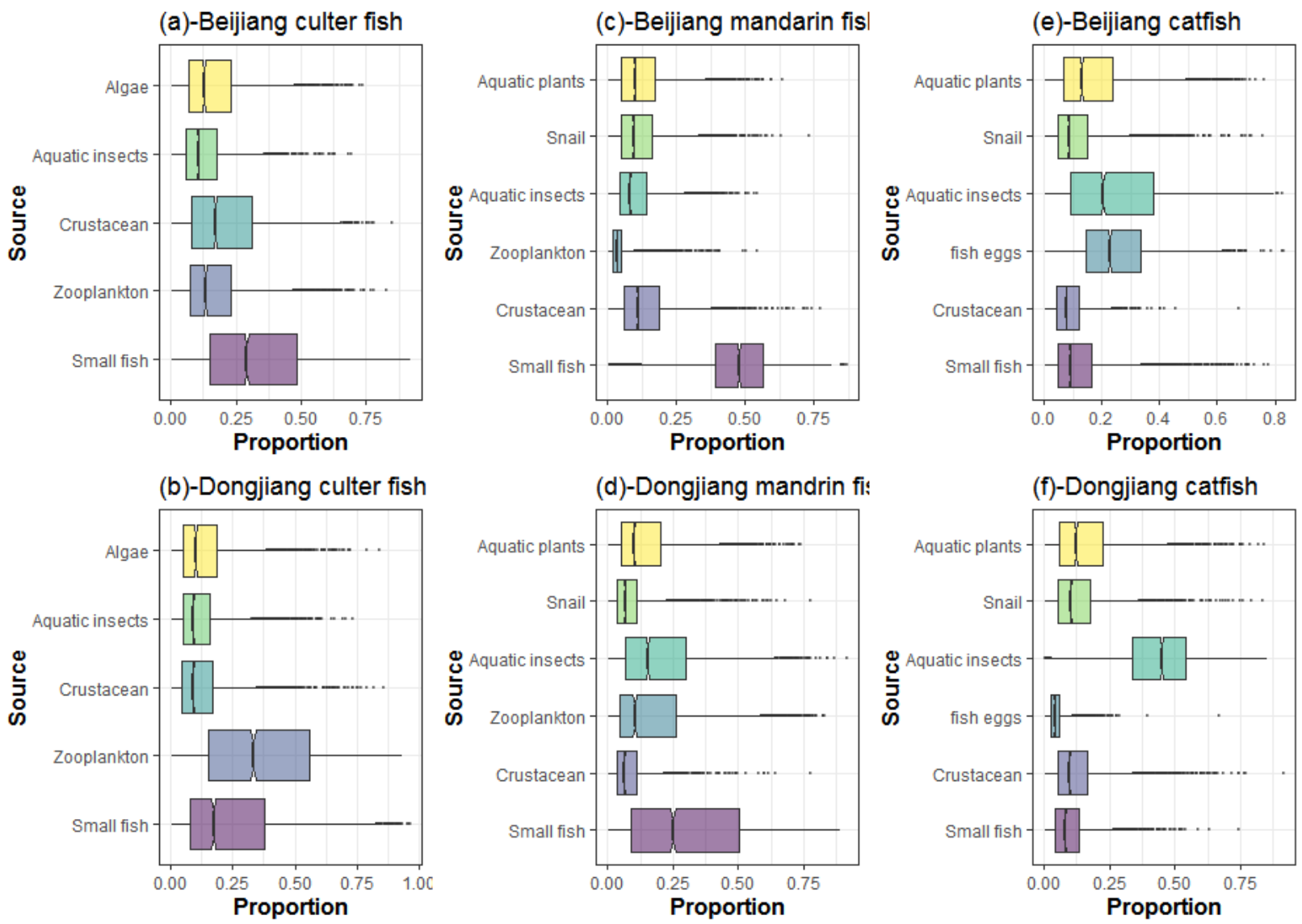

Figure 5

Food resource structure of uninvaded and invaded rivers.

\section{Supplementary Files}

This is a list of supplementary files associated with this preprint. Click to download.

- AppendixTableS1.docx 